\title{
Marina Yaguello (dir.), Le grand livre de la langue française
}

\section{Ruggero Druetta}

\section{Q OpenEdition}

1 Journals

\section{Édition électronique}

URL : https://journals.openedition.org/studifrancesi/38557

DOI : 10.4000/studifrancesi.38557

ISSN : 2427-5856

Éditeur

Rosenberg \& Sellier

\section{Édition imprimée}

Date de publication : 15 décembre 2004

Pagination : 672-674

ISSN : 0039-2944

\section{Référence électronique}

Ruggero Druetta, "Marina Yaguello (dir.), Le grand livre de la langue française », Studi Francesi [En ligne], 144 (XLVIII | III) | 2004, mis en ligne le 30 novembre 2015, consulté le 08 mai 2021. URL : http:// journals.openedition.org/studifrancesi/38557 ; DOI : https://doi.org/10.4000/studifrancesi.38557

Ce document a été généré automatiquement le 8 mai 2021.

\section{(c) $(1) \&$}

Studi Francesi è distribuita con Licenza Creative Commons Attribuzione - Non commerciale - Non opere derivate 4.0 Internazionale. 


\title{
Marina Yaguello (dir.), Le grand livre de la langue française
}

\author{
Ruggero Druetta
}

\section{RÉFÉRENCE}

MARINA YAGUELLO (dir.), Le grand livre de la langue française, Paris, Seuil, 2003, pp. 564.

«Ni manuel, ni ouvrage de référence» (p. 9), le volume dirigé par M. Yaguello fait le point sur le français au début du XXI ${ }^{\mathrm{e}}$ siècle et sur sa place dans le monde. Réunissant plusieurs linguistes de renom, l'ouvrage est organisé en monographies autonomes qui offrent chacune un aperçu descriptif des problématiques envisagées, sans renoncer pour autant à poser des jalons explicatifs. La rigueur de l'approche, alliée à un langage sobre, à la multiplication des exemples concrets ainsi qu'à l'utilisation de nombreuses cartes et tableaux, rend la lecture accessible aux spécialistes comme aux nonspécialistes. Le volume est complété par un index, alors que la bibliographie est répartie au terme de chaque monographie.

«Le français dans l'histoire» (Christiane MARCHELLO-NIZIA, pp. 11-90), après avoir situé le français dans le panorama des langues romanes, retrace les grandes étapes de son évolution externe et interne, sans oublier des aspects complémentaires comme l'évolution des graphies. Les textes cristallisant l'état de la langue à une période donnée sont analysés minutieusement: c'est le cas des Serments de Strasbourg, de la Chanson de Roland, d'une Ballade de Villon, d'une page du Journal d'Hérouard, de passages de Fénelon, Hugo ou Camus entre autres. Les changements dans le système de la langue sont regroupés autour des fonctions syntaxiques majeures (groupe nominal, groupe verbal, phrase et structure informationnelle): on passe donc des faits morphologiques les mieux connus (perte de la déclinaison, évolution des déterminants, enclise...) aux questions concernant l'ordre de mots (place du sujet, constructions disloquées, constructions clivées, propositions interrogatives...). Les tendances les plus récentes sont également prises en considération: changements dans les prépositions, emploi 
adverbial des adjectifs, grammaticalisations. La dernière partie est consacrée à l'évolution du système phonologique.

3 «La variation: le français dans l'espace social, régional et international» (Françoise GADET, pp. 91-152) met à mal la prétendue homogénéité du français, dont la sociolinguistique a montré la diversité irréductible des usages. L'A. commence par un aperçu de la francophonie, qui distingue entre pays où le français est langue maternelle, langue seconde ou langue véhiculaire, ce qui est à la base d'usages différents; ensuite elle rappelle les grands axes théoriques de la variation linguistique, dont elle donne des exemples francophones. Certains procédés typiques du français sont également évoqués (argot, verlan, javanais), avant d'en arriver au problème de fond de la norme et de la standardisation du français et du rapport des français vis-àvis de celle-ci, sans oublier le jugement qu'entraine la perception de toute forme de variation/déviance chez les locuteurs (cris d'alarme réguliers contre une «crise du français», préjugés sur les façons de parler, insécurité et hypercorrections). Les problèmes de covariance et de dynamique entre les différents ordres de variations sont également abordés, avec la focalisation de quelques tendances plus marquées, comme les tranches d'âge, le sexe ou l'appartenance sociale pour ce qui concerne la variation diastratique. La variation diatopique est abordée sous l'angle complémentaire de la variation régionale, à l'échelle française, et de la variation francophone, à l'échelle internationale. C'est dans le prolongement de cette analyse que se trouvent des réflexions sur les situations de diglossie et les phénomènes de créolisation, alors que la présentation des différents volets de la politique linguistique du français vient conclure le tour d'horizon des variétés du français, confirmant son caractère de langue d'état. «La grammaire» (Marina YAGUELLO, pp. 153-258) entreprend la description des structures de la langue. La description ne suit pas l'ordre traditionnel des grammaires scolaires (les parties du discours), mais s'organise autour des trois pôles qui façonnent la structure de l'énoncé: la pragmatique, la sémantique et la syntaxe. L'A. s'appuie, dans sa présentation, sur les résultats de la linguistique de grands corpus, qui permettent un meilleur éclairage des phénomènes par la mise en perspective de leur fréquence. Le volet pragmatique classe d'abord les modalités de phrase et les formes verbales qui les caractérisent à partir des actes de langage qu'elles sont à même de réaliser. Les verbes de parole, les performatifs délocutifs et les actes de langage indirects y tiennent une place centrale. L'A. passe ensuite à la structure informationnelle des énoncés, avec l'inventaire des structures permettant la gestion du thème et du focus. L'analyse du composant sémantique touche d'abord des notions à la réalisation multiforme telles que le temps grammatical, l'aspect ou la modalité, qui peuvent avoir une réalisation tantôt morphologique, tantôt lexicale. Sont ensuite traitées les catégories de détermination, de genre et de nombre, permettant d'appréhender de nombreux aspects de la morphologie nominale. C'est ensuite le plan de l'énoncé qui est analysé du point de vue de ses propriétés sémantiques, introduisant les notions de factivité, de généricité/spécificité, de sens propre/figuré. L'A. conclut cette partie par la mise en évidence de la différence entre le plan sémantique et le plan syntaxique dans le cas de l'analyse des rôles sémantiques des actants de l'énoncé. L'analyse du composant syntaxique opère une distinction préliminaire entre grammaire de la phrase (au statut pourtant controversé) et grammaire du texte, puis essaie de définir les notions utilisées (phrase simple, complexe, constituant, valence verbale, classe syntaxique - avec tout le problème des membres prototypiques des différentes classes et du gradient d'une classe à l'autre). Sont ensuite traités les cas d'interaction entre les trois composants, 
que l'A. avait déjà évoqués précédemment, avec une analyse plus détaillée et des exemples plus abondants: les outils linguistiques de la thématisation et de la focalisation (pour l'interaction syntaxe-pragmatique) et ceux qui signalent un sens figuré ou la possession inaliénable (pour l'interaction syntaxe-sémantique). Après cette analyse de la syntaxe, l'A. aborde le domaine de la morphologie dérivationnelle et flexionnelle (nominale et verbale), dont l'importance dans les manuels scolaires est motivée par le purisme et le très grand écart qui sépare la morphologie de l'oral de la morphologie de l'écrit en français; à signaler la présence de tableaux statistiques détaillant l'étendue des différents morphèmes de genre ou la fréquence relative des verbes irréguliers. L'exposé se termine par la grammaire de texte, dont l'A. analyse les notions, en commençant par tout ce qui contribue à en déterminer la texture (cohésion, cohérence, intentionnalité, informativité, intertextualité, présence de bornes textuelles); cette présentation touche ensuite les plans d'énonciation et se termine sur les outils de cohésion que sont l'anaphore et la coréférence.

5 La phonologie est traitée dans «Les sons du français» (Bernard TRANEL, pp. 259-315). Après un rappel des fonctions de l'orthographe vis-à-vis de la prononciation, l'A. aborde successivement l'écart entre graphèmes et phonèmes en français, la notation API, ainsi que les principales notions de phonétique articulatoire, qui constituent le préalable à l'introduction de la notion de phonème et de paire minimale. L'inventaire des voyelles met en évidence la permanence de quelques diphtongues phonologiques permettant par exemple d'expliquer la liaison dans les oiseaux et pas avec les waters, phoniquement identiques; parmi les consonnes, le cas de ' $r$ ' et de ses variantes est passé au peigne fin. L'analyse se déplace ensuite au niveau de la syllabe, dont l'A. présente d'abord la structure, qui lui sert de base pour évaluer la distribution caractéristique des sons dans chaque langue ainsi que la distribution particulière des voyelles moyennes en français, dont l'opposition d'ouverture se trouve neutralisée suivant que la syllabe est fermée ou ouverte. D'autres facteurs, comme l'harmonie vocalique ou l'influence de la morphologie, peuvent également jouer un rôle. Sont ensuite passés en revue d'autres points sensibles de la phonologie du français: l'opposition entre 'a' antérieur et postérieur, le système des voyelles nasales (l'A. ne mentionne que trois termes, sans discuter le statut de 'un'), les sons «à éclipse», parmi lesquels le schwa occupe une place centrale, et le sujet de la liaison, sans oublier les phénomènes d'hypercorrection représentés par les liaisons fautives.

6 «La langue parlée» (Claire BLANCHE-BENVENISTE, pp. 317-344) décrit la différence des mécanismes linguistiques sur lesquels s'appuient le français parlé et le français écrit. Après avoir écarté l'hypothèse de la diglossie et avoir présenté les outils techniques ayant permis une approche rigoureuse de l'oral, l'A. passe en revue les préjugés ordinaires affectant la langue parlée, puis justifie la nécessité de la transcription écrite assortie de notations prosodiques et phonétiques pour éviter l'impasse des analyses impressionnistes. La prise en compte de grands corpus montre qu'il y a un certain nombre de «fautes» banales, produites par tous les locuteurs, quel que soit leur niveau d'instruction, comme les simplifications phonétiques, l'absence du ne de négation ou du sujet $i l$ avec certains verbes impersonnels. L'A. expose ensuite les particularités de la grammaire de la langue parlée, où elle essaie de montrer que la moindre présence de marques morphologiques n'est pas le synonyme d'absence de grammaire. Les marques de genre et de nombre, non audibles dans la plupart des mots, sont étalées au niveau du syntagme nominal, et les «fautes» de liaison au pluriel (machines à laver - $z$ - italiennes) sont souvent une manière de signaler le nombre à l'oral. La morphologie verbale est 
également abordée sous l'angle de sa moindre richesse par rapport à l'écrit et des régularisations pratiquées par les locuteurs. Dans la partie consacrée à la syntaxe, l'A. souligne combien les différences relevées entre les productions écrites et orales (répétitions, énoncés clivés ou pseudo-clivés, dislocations, dilution de l'information, parataxe) répondent en fait à des règles rédactionnelles (donc apprises), alors que certaines constructions plus spécifiques (par ex. le dispositif il y a...qui pour introduire un sujet indéfini, l'antéposition de compléments valenciels, l'hypotaxe sans marqueurs) font généralement l'objet d'une réprobation normative.

«L'orthographe» (Claire BLANCHE-BENVENISTE, pp. 345-389) propose une réflexion sur les principes linguistiques et extralinguistiques qui régissent le «supplément orthographique» de l'écriture de la langue. Après avoir montré les déficits de l'alphabet latin et les solutions adoptées par le français pour les pallier (diacritiques, di- et trigrammes, non sans rappeler que l'usage de ces derniers a parfois déterminé l'entrée dans la prononciation de lettres non étymologiques), l'A. démontre l'impraticabilité d'une écriture phonographique du français à partir de l'alphabet latin. Le fonctionnement non univoque des lettres diacritiques (sont-elles à considérer comme de vraies lettres ou comme de simples diacritiques?) est épinglé, tout comme les différences de prononciation régionales, qui empêchent de s'appuyer sur la prononciation pour justifier telle ou telle orthographe. Si les exigences des scripteurs justifient qu'on réclame une réforme de l'orthographe, le point de vue du lecteur, qui identifie les mots à partir de leurs particularités graphiques, s'y oppose fermement. Il s'agit des fonctions lexicales et grammaticales de l'orthographe, comme l'identification d'une famille de mots à travers la stabilité graphique du radical, le marquage de l'étymologie (même si c'est loin d'être systématique) - qui a son pendant dans les nombreuses étymologies populaires, la distinction des homophones, les marques de variabilité et d'accord (genre, nombre, personne). Le problème de l'accord verbal, et notamment du participe passé, est également soumis à une analyse critique. L'exposé se termine sur les réformes orthographiques, dont la dernière en date est présentée dans le détail et dont on montre néanmoins les contradictions.

8 «Le lexique» (Jean-Paul colin, pp. 391-456) commence par discuter la notion préthéorique de mot, avant d'introduire les notions de base de la lexicologie (structuration du lexique, champ, sémasiologie, onomasiologie, hypo- / hyperonymie), appliquées au vocabulaire français. L'étude de la morphologie lexicale est consacrée aux sources du lexique (indigènes et d'emprunt), avec le corollaire des catégories d'emprunt et des différentes sortes d'adaptation phonologique et graphique de ceux-ci. L'étude diachronique est complétée par la description du changement lexical (déformation et remplacement de mots, néologismes et leur pendant, les archaïsmes et autres mots désuets), dont l'A. analyse les causes externes et internes; il y analyse, entre autres choses, la néologie du Web ainsi que les tendances du vocabulaire branché ou «politiquement correct». L'étude synchronique comprend les différents phénomènes de dérivation ainsi que leur fonction: l'A. part de l'affixation, dont il étudie également la productivité des affixes, puis se penche sur la composition et ses contraires, la troncation et la siglaison. Les caractéristiques des locutions sont également prises en compte, ainsi que leur éventuel caractère idiomatique. La dernière partie de l'exposé est consacrée à la sémantique lexicale, avec la position des concepts de base (désignation, dénotation, connotation) ainsi que des relations entre lexèmes (synonymie, parasynonymie, paronymie). La notion de registre est enfin convoquée comme clé d'accès aux langues de spécialités, alors que le tabou linguistique est à la 
base d'usages lexicaux aussi variés que le contournement (périphrase et euphémismes) ou le cryptage (argot).

«Les dictionnaires français: histoires et méthodes» (Jean PRUVosT, pp.457-489) retrace la genèse, puis l'histoire des dictionnaires français, montrant à la fois la place centrale qu'ils ont vite assumé dans les pratiques (en 1997,82\% des foyers possédaient un dictionnaire) et dans l'imaginaire symbolique de la nation (au point que Quillet, en 1934, le considère comme une Bible laïque dépositaire de la connaissance). L'A. s'attache aussi à démontrer combien cet objet, souvent idolâtré comme l'autorité linguistique absolue «est-ce dans le dictionnaire?»), est en réalité soumis aux impératifs de l'époque et aux moyens techniques à la disposition de ses auteurs. L'exposé historique part des glossaires du Moyen-Âge et des dictionnaires bilingues pour arriver au premiers monolingues ou faux bilingues (R. Estienne et J. Nicot), puis focalise la trilogie fondatrice du Grand Siècle, avec les ouvrages de Richelet, prototype du dictionnaire de langue, de Furetière, qui ouvre la voie des dictionnaires encyclopédiques, et de l'Académie, premier dictionnaire institutionnel fondé sur l'usage courant. L'A. retrace ensuite l'évolution de ces trois typologies, avec notamment l'Encyclopédie au XVIII ${ }^{e}$ siècle, les dictionnaires historiques du XIX ${ }^{e}$, ainsi que les débuts des dictionnaires commerciaux comme celui de Larousse, et la création des dictionnaires réduits, condensés à partir des éditions majeures, pour un public scolaire de plus en plus étendu. De ce point de vue, le $\mathrm{XX}^{\mathrm{e}}$ siècle sera celui des dictionnaires d'apprentissage, spécifiquement conçus pour le public auquel ils sont destinés. Les grands dictionnaires des deux siècles derniers (Littré, Larousse, Hatzfeld et Darmesteter, Robert, TLF) sont analysés dans leurs fondements épistémologiques et lexicographiques, avant de conclure sur les nouveaux médias qui modifient radicalement l'approche à ces outils et sur les développements théoriques récents, qui poussent l'A. à exploiter la trilogie lexicographie - dictionnairique - métalexicographie (B. Quemada) pour aborder une «science des dictionnaires».

10 «Enseigner la langue française maternelle» (Émile GENOUVRIER, pp. 491-541) examine les aspects pédagogiques et législatifs de l'enseignement du français en milieu scolaire, de la maternelle au collège. L'A. aborde les différentes forces qui (sur)déterminent cet enseignement: caractéristiques intrinsèques du système, évolution des modèles linguistiques et didactiques, pratiques historiquement ancrées, pressions et mutations sociales, représentations collectives... sans oublier les caractéristiques évolutives des apprenants, dont il reproduit souvent les productions afin de montrer les écueils et les enjeux des multiples compétences convoquées par l'apprentissage du français langue maternelle. L'exposé est en effet organisé autour des composantes de la compétence communicative: parler (avec le problème de la parole en classe), lire (quels sont les modèles d'accès à la reconnaissance du mot, pourquoi et comment lire), écrire (apprentissage de nouveaux mots, gestion du discours écrit, par exemple dans la rédaction, exercice traditionnel de l'école française), connaitre la langue (avec tous les problèmes que pose l'apprentissage et l'emploi d'un métalangage déterminé et le bienfondé remis en cause de certaines approches entérinées par la tradition), l'orthographe (dont il explique minutieusement et sans préjugé les achoppements, mais aussi les atouts, que ce soit au niveau du mot isolé ou de la phrase, ce qui aboutit à prôner un enseignement ni idolâtre ni laxiste de cette discipline), la grammaire (dont il critique les finalités qui lui sont souvent assignées), le lexique (qui demande un travail quantitatif et qualitatif à la fois). 